\title{
The role of temporal cue-target overlap in backward inhibition under task switching
}

\author{
Michel D. Druey ANd Ronald HÜBNeR \\ Universität Konstanz, Konstanz, Germany
}

\begin{abstract}
One of the proposed mechanisms for sequential control in task-shift conditions is backward inhibition (BI), which is usually measured in terms of lag-2 task repetition costs in A-B-A task sequences relative to C-B-A task sequences. By considering the so far existing experiments it seems that these lag- 2 repetition costs occur only with temporally overlapping cues and targets. In the present study this issue was further examined in two experiments, in which temporal cue-target overlap was varied blockwise (Experiment 1) and from trial-to-trial (Experiment 2). Experiment 1 shows that lag-2 repetition effects can only be observed with temporally overlapping cues and targets, indicating that there was no BI with temporally separated cues and targets. However, the results of Experiment 2 suggest that both irrelevant task sets are inhibited in this case, and that with temporally overlapping cues and targets only the previously relevant task set is inhibited.
\end{abstract}

Recently, the task-shift paradigm has widely been applied to investigate the mechanisms of mental control. In corresponding experiments, participants have to shift between different tasks across trials (e.g., Mayr \& Kliegl, 2003; Rogers \& Monsell, 1995), and several mechanisms have been proposed, which seem to be involved in performing such shifts (see, e.g., Monsell, 2003). One of these mechanisms is the suppression of the previously relevant task set if shifting to a new task set is required. This socalled backward inhibition (BI) has first been studied by Mayr and Keele (2000), and is generally thought to reduce interference from the just abandoned task set (cf. Arbuthnott, 2005; Hübner, Dreisbach, Haider, \& Kluwe, 2003).

In order to investigate BI, Mayr and Keele (2000) applied a task-shift paradigm, in which shifting among three different tasks (A, B, and $\mathrm{C}$ ) was required, and compared performance in A-B-A task sequences with that in C-B-A sequences. If the previously relevant task set is inhibited when shifting to task $\mathrm{B}$ on trial $n-1$, and if part of this inhibition is still in effect when a shift back to task A on trial $n$ is required, then performance on trial $n$ in the A-B-A sequences should be impaired relative to that in the C-B-A sequences. In other words, there should be lag-2 repetition costs (or alternating-switch costs, cf. Arbuthnott \& Woodward, 2002). Indeed, such costs have been observed by Mayr and Keele (2000) as well as in numerous subsequent studies (e.g., Arbuthnott \& Frank, 2000; Dreher \& Berman, 2002; Dreher, Kohn, \& Berman, 2001; Gade \& Koch, 2005; Mayr, 2002; Mayr, Diedrichsen, Ivry, \& Keele, 2006; Mayr \& Kliegl, 2003; Schuch \& Koch, 2003).

In contrast, though, our own attempts to find lag-2 repetition costs in several preliminary experiments were less successful, even though we applied a procedure, which was similar to that in other studies (e.g., Schuch \& Koch, 2003). However, a closer look revealed that in all studies reporting lag- 2 repetition costs, the cues remained present during target presentation, whereas they disappeared before target onset in our preliminary experiments. This suggests that cues and their temporal relation to the target play a crucial role for BI. That cues and their properties modulate the lag- 2 repetition effects has recently been shown by Arbuthnott (2005). In her study, different cue-types (e.g., spatial vs. symbol cues) affected the lag-2 repetition costs differentially (e.g., larger costs for symbol than for spatial cues). Therefore, our main aim in the present study was to examine whether presenting cues and targets temporally overlapping is indeed crucial for obtaining lag-2 repetition effects.

\section{EXPERIMENT 1}

In Experiment 1, we compared performance in a condition with temporally overlapping cues and targets (i.e., the standard cuing condition) with that in a condition where the cues and targets appeared temporally separated in a blocked design. If temporal cue-target overlap ${ }^{1}$ is indeed crucial for BI, then the usual lag-2 repetition costs should occur under this condition. In contrast, no such costs are expected if the cues disappear before target onset.

In addition to temporal cue-target overlap, we also varied the relative positions of cues and targets. The reason for this variation was that, in some studies, cues and targets were presented spatially integrated (e.g., Dreher \& Berman, 2002; Schuch \& Koch, 2003), whereas in other studies they appeared spatially separated (e.g., Arbuth- 

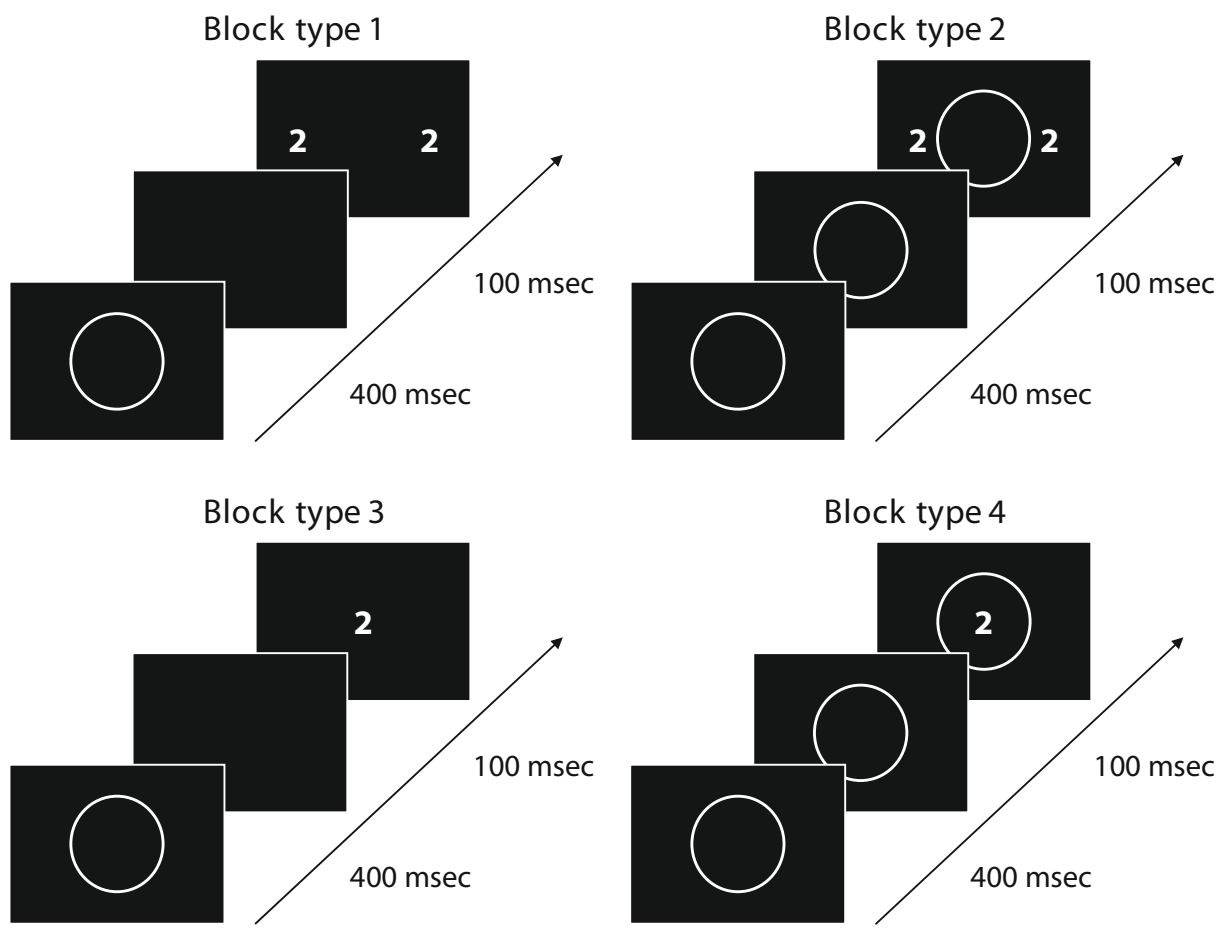

Figure 1. Schematic depiction of the presentation sequences from cue onset to response execution for each of the four block types in Experiment 1.

nott \& Woodward, 2002; Mayr et al., 2006). Although it is unlikely that this factor alone affects the lag-2 repetition costs, it can not be excluded that it eventually interacts with temporal cue-target overlap.

\section{Method}

Participants. Sixteen students ( 12 female; mean age $=28.2$ years $)$ from the Universität Konstanz participated in this experiment.

Apparatus and Stimuli. Stimulus presentation and response recording were controlled by an IBM-compatible PC. The stimuli were presented on a 21-in. color monitor with a resolution of 1,280 $\times 768$ pixels and a refresh rate of $85 \mathrm{~Hz}$.

The digits 1 to 4 and 6 to 9 served as targets. They appeared in white on a black background at the center of the screen, each subtending a visual angle of $1.99^{\circ}$ in height and approximately $1.27^{\circ}$ in width at a viewing distance of $100 \mathrm{~cm}$.

Procedure. Participants had to judge either parity (odd/even), magnitude (less/greater than 5) or position on the number line from 1 to 9 (central $[3,4,6,7]$ / peripheral $[1,2,8,9]$ ) of the target digit. There were only task shift trials.

Two buttons, pressed with the index and middle fingers of the right hand, respectively, served as response keys. "Even," "less," and "central" were mapped to the left button, and "odd," "greater," and "peripheral" to the right button, respectively.

Each trial started with the presentation of a cue at the center of the screen, which indicated the relevant judgment type. They could have one of three forms: (1) a square indicating the parity judgment, (2) a diamond indicating the magnitude judgment, and (3) a circle indicating the position judgment. The cues subtended a visual angle of about $3.64^{\circ} \pm 0.62^{\circ}$ in width.

There were four different block-types regarding cue and target presentation (see Figure 1). (1) The cue appeared centrally on the screen for $400 \mathrm{msec}$ and disappeared $100 \mathrm{msec}$ before target onset. Two copies of the target were then presented to the left and right of the center of the screen with an eccentricity of about $2.58^{\circ}$. (2) The cue appeared centrally and remained present until response execution. Two copies of the target appeared laterally as in the first block type $500 \mathrm{msec}$ after cue onset. (3) Cues and targets were both presented centrally but the cue disappeared again after $400 \mathrm{msec}, 100 \mathrm{msec}$ before target onset. (4) Both cues and targets were presented centrally with the target appearing $500 \mathrm{msec}$ after cue onset within the cue. They remained present until the participants responded. In all four conditions, the stimuli were replaced by a blank screen for $1,000 \mathrm{msec}$ immediately after responding and until presentation of the next cue.

Each participant performed a total of 8 practice blocks ( 2 of each type) with 48 trials each and 16 experimental blocks ( 4 of each type) with 96 trials each at two sessions. Blocks were organized into sequences of four (one of each type) and block-order within these sequences was counterbalanced across participants.

\section{Results}

Response times (RT) and error rates (ER) were analyzed in separate three-way repeated-measures ANOVAs considering the factors sequence type (A-B-A, C-B-A), temporal cue-target overlap (overlapping, separated), and spatial cue-target integration (integrated, nonintegrated).

RT. The analysis revealed significant main effects of sequence type $[F(1,15)=9.66, p<.01]$, reflecting overall lag-2 repetition costs of $20 \mathrm{msec}$, and of temporal overlap $[F(1,15)=25.12, p<.001]$, reflecting faster responses in conditions with temporally separated cues and targets (501 $\mathrm{msec}$ ) than in conditions with temporally overlapping cues and targets $(529 \mathrm{msec})$. However, also the interaction between sequence type and temporal overlap was significant $[F(1,15)=12.22, p<.01$; see Figure 2]. Further analyses revealed that the lag- 2 repetition costs were reliable only for temporally overlapping cues and targets [28 msec, 
$F(1,15)=19.69, p<.001]$, but not for temporally separated ones $[12 \mathrm{msec}, F(1,15)=2.66, p>.12]$. Furthermore, the interaction between sequence type and spatial integration was also significant $[F(1,15)=8.44, p<.05]$. Similarly, as for temporal overlap, reliable lag-2 repetition costs occurred only for spatially integrated cues and targets [34 msec, $F(1,15)=10.99, p<.01]$, but not for spatially nonintegrated ones [6 msec, $F(1,15)=1.62, p>.22]$.

ER. There were significant main effects of temporal overlap $[F(1,15)=7.29, p<.05]$, and of spatial integration $[F(1,15)=5.48, p<.05]$. The participants made more errors for temporally separated $(6.91 \%)$ than for temporally overlapping $(6.19 \%)$ cues and targets (see Figure 2). They also made more errors for spatially nonintegrated $(6.94 \%)$ than for spatially integrated $(6.17 \%)$ cues and targets. Furthermore, the interaction between sequence type and spatial integration was also significant $[F(1,15)=7.38, p<.05]$. If the cues and targets were presented spatially nonintegrated, the participants made more errors in the A-B-A task sequences than in the C-B-A task sequences ( $7.37 \%$ vs. $6.50 \%$, respectively). This pattern was reversed for spatially integrated cues and targets (5.91\% vs. $6.42 \%$, respectively).

\section{Discussion}

Our results show that, with respect to the lag-2 repetition costs, it is indeed crucial whether the cues and targets appeared temporally overlapping or separated (see Figure 2). Whereas reliable costs occurred for temporally overlapping cues and targets, no such costs could be observed for temporally separated ones. Regarding the RTs, a similar effect also occurred for the spatial integration of cues and targets, but there was an opposite effect for the ERs, which indicates a speed-accuracy trade-off. Moreover, there was no reliable interaction between temporal overlap and spatial integration. Therefore, in the following, we focus exclusively on the effects of temporal cue-target overlap.

According to the standard BI-account, the fact that there was no lag-2 repetition effect for task sequences with temporally separated cues and targets suggests that, under this condition, the previously applied task set was not inhibited if a task shift was required. One possible explanation for this noninhibition may be that task performance was generally easier with temporally nonoverlapping cues and targets, as indicated by the faster responses in the nooverlap condition. However, as we used a blocked design (i.e., the temporal overlap of cues and targets was confounded throughout the individual trails $n-2, n-1$, and $n$ of the respective A-B-A and C-B-A task sequences), we have to be careful with interpreting the absolute performance differences between the two conditions. Thus, in order to examine why there was no lag-2 repetition effect in the no-overlap condition, the trial-specific effects of temporal cue-target overlap on BI must be revealed. This was done in Experiment 2.

\section{EXPERIMENT 2}

In this experiment, the temporal overlap of cues and targets was varied across trials. This should allow us to compare task sequences, which differ only with respect to the temporal cue-target overlap on one of the trials in the respective task sequences. With this procedure, we should be able to determine at which position in the trial sequence the effects of temporal cue-target overlap affect the lag-2 repetition effects.

\section{Method}

Participants. Sixteen new students $(11$ female; mean age $=$ 24.4 years) from the Universität Konstanz participated in this experiment.

Apparatus, Stimuli, and Procedures. The apparatus, the stimuli, and the tasks were the same as in Experiment 1. Also the procedure was similar, except that temporal cue-target integration varied from trial-to-trial. Moreover, spatial cue-target integration was not considered here. Rather, the targets appeared always at fixation.

Each participant performed 14 blocks of 48 trials each. The first two blocks were considered as practice blocks and were therefore not included in the analyses.

\section{Results}

RTs and ERs were analyzed individually in four-way repeated-measures ANOVAs with sequence type (A-B-A, C-B-A), temporal cue-target overlap on trial $n-2$ (overlapping, separated), temporal cue-target overlap on trial $n-1$ (overlapping, separated), and temporal cue-target overlap on trial $n$ (overlapping, separated) as relevant factors.

RT. The analysis revealed a significant main effect of sequence type $[F(1,15)=12.24, p<.01]$, reflecting overall lag-2 repetition costs of $24 \mathrm{msec}$. Also the main effects of temporal cue-target overlap on trial $n-2[F(1,15)=$ $17.29, p<.001]$ and on trial $n[F(1,15)=32.74, p<$ $.001]$ were reliable. Regarding the overlap on trial $n-2$, the participants responded faster on the present trial in the overlapping condition $(579 \mathrm{msec})$ than in the separated

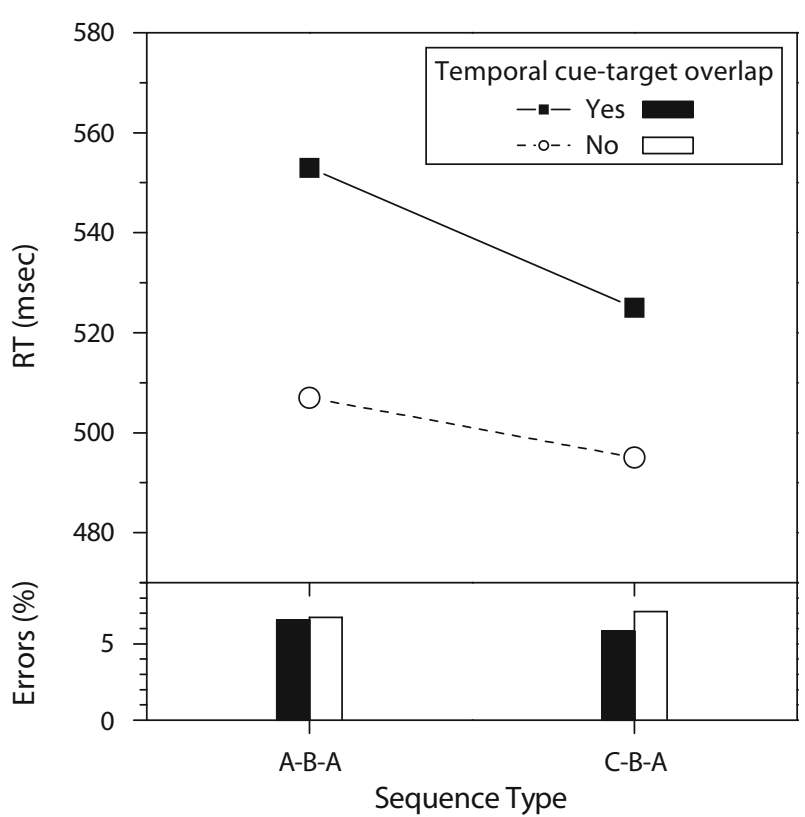

Figure 2. Response times and error rates in Experiment 1 as functions of sequence type and temporal cue-target overlap. 


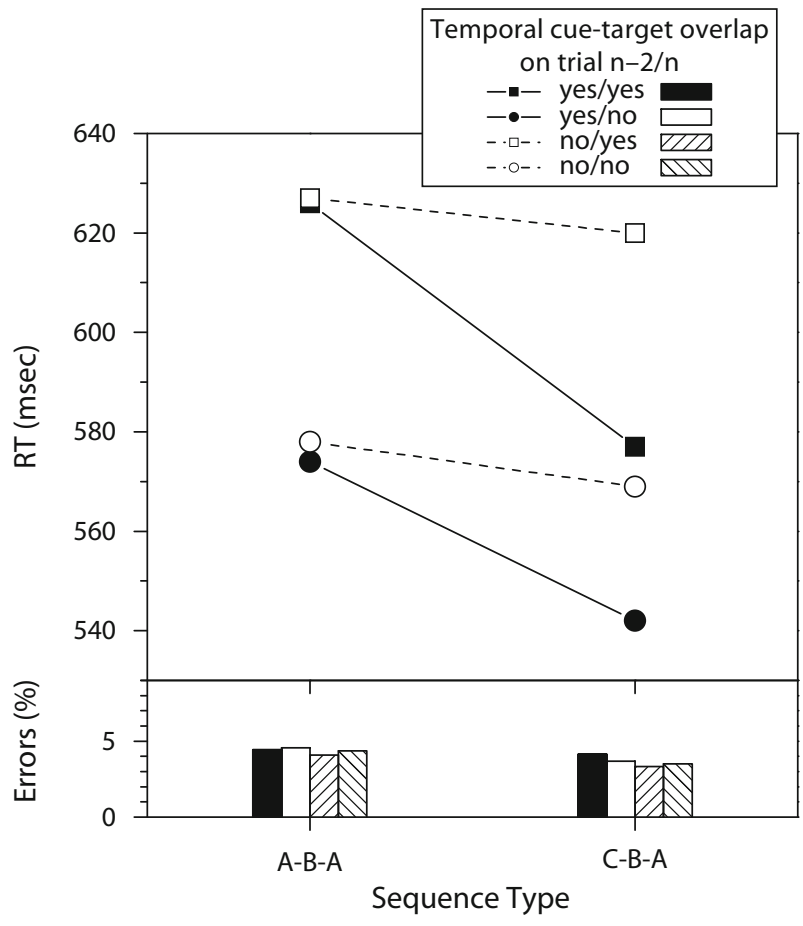

Figure 3. Response times and error rates in Experiment 2 as functions of sequence type and temporal overlap of cues and targets on trials $n-2$ and $n$.

condition (599 msec). This relation was reversed, though, for temporal cue-target overlap on trial $n$ (613 msec vs. $566 \mathrm{msec}$ ). Furthermore, the two-way interaction between sequence type and overlap on trial $n-2$ was significant $[F(1,15)=9.42, p<.01]$, which indicates that the lag-2 repetition costs were considerably larger for overlapping [40 msec, $F(1,15)=26.99, p<.001]$ than for separated cues and targets on trial $n-2[8 \mathrm{msec}, F(1,15)=.69, p>$ .41 ; see Figure 3 ]. The main effect of temporal overlap on trial $n-1$ as well as the interactions between sequence type and overlap on trials $n-1$ and $n$ were not reliable $(F \mathrm{~s}<1, p \mathrm{~s}>.57)$.

ER. The overall mean ER was rather low (4.01\%), and there were no significant effects.

\section{Discussion}

As in our first experiment, lag-2 repetition costs occurred only for temporally overlapping cues and targets. Moreover, as the trial-specific analysis revealed, only the overlap on trial $n-2$ was crucial in this respect. Neither an overlap on trial $n-1$ nor one on trial $n$ had any reliable effect on the lag-2 repetition costs. However, as can be seen in Figure 3 (squares vs. circles), the participants generally responded faster if there was no cue-target overlap on trial $n$. Several mechanisms could be responsible for this effect. For instance, the forewarning of stimulus onset could be more precise in the no-overlap condition. Furthermore, in this condition, there was also no need to divide attention between cue and target, which could have been an advantage. Finally, if the cue remained present during target presentation, this could have induced addi- tional rechecking processes in order to select the correct task set. In any case, the present result confirms our suspicion that the absolute performance difference between the two blocked overlap conditions in Experiment 1 can not seriously be interpreted in terms of task-set inhibition.

In contrast, the result that a cue-target overlap on trial $n-2$ produced costs for A-B-A sequences relative to C-B-A sequences (see the filled symbols in Figure 3) can easily be interpreted as indicating BI: The task set for task A was inhibited on trial $n-1$ in this case. Thus, if the participants had to return to task A on trial $n$ in A-B-A sequences, they had to overcome the residual inhibition associated with this task, which produced costs. The crucial question now is why there was no performance difference for the two task sequence types when there was no cue-target overlap on trial $n-2$.

First, without temporal cue-target overlap on trial $n-2$, no BI could have taken place on trial $n-1$. As a consequence, the task set for task A should have been in the same uninhibited state on trial $n$ for both sequence types. Accordingly, no performance differences should occur for these sequence types, which is indeed what we observed. However, it also follows from these assumptions that the performance should be the same as that for the C-B-A sequences in the overlap condition, which was obviously not the case. Rather, performance was as that for the A-B-A sequences. The prediction is even worse, if we further assume that there was not only no BI in the no-overlap condition but positive task-set priming from trial $n-2$ to $n$. In this case, the performance should even have been better in the A-B-A than in the C-B-A sequences.

But how else can our data for the no-overlap condition be explained? A straightforward account would be to assume that $\mathrm{BI}$ is not selective on trial $n-1$ when the cues and targets appeared temporally separated on trial $n-2$. Thus, both irrelevant task sets on trial $n-1$ (i.e., A and $C$ ) are inhibited. In this case, the performance should not differ between the C-B-A and the A-B-A sequences, because the task set for task $A$ is in an inhibited state for both sequence types. Moreover, the performance should be similar to that for the A-B-A sequences in the $n-2$ overlap condition, which is exactly what we observed (see Figure 3, filled vs. open symbols).

\section{GENERAL DISCUSSION}

Our primary aim in this study was to examine the effects of temporal cue-target overlap on the lag-2 repetition costs, which are usually interpreted in terms of task-set inhibition. In all studies reporting such costs so far, the cues remained present during target presentation (e.g., Mayr \& Keele, 2000; Schuch \& Koch, 2003), whereas no such costs occurred in our own preliminary experiments, where the cues disappeared before target onset. In Experiment 1, we therefore varied temporal cue-target overlap blockwise in order to contrast the standard cuing procedure with one in which the cues and targets were temporally separated. As expected, reliable lag- 2 repetition costs occurred only with the standard cuing procedure. This suggests that a temporal cue-target overlap is essential for BI. 
However, due to the blocked design in Experiment 1, it was not possible to determine which task-sequence position was essential regarding the effects of temporal cue-target overlap on the lag- 2 repetition costs. Therefore, in Experiment 2, temporal cue-target overlap was varied across trials. The trial-specific analysis revealed that a cue-target overlap was crucial only on trial $n-2$. Reliable lag- 2 repetition costs on trial $n$ occurred only if there was a temporal cue-target overlap on trial $n-2$. If the cues and targets did not overlap temporally on trial $n-2$, then there was no performance difference between the A-B-A and C-B-A task sequences. Moreover, the RTs under this condition were similar to those for A-B-A sequences in the $n-2$ overlap condition.

This pattern of results can straightforwardly be explained if one assumes that with temporally separated cues and targets on trial $n-2$, both irrelevant task sets on trial $n-1$ were inhibited. In this case, the task set for task $A$ is in the same state of inhibition irrespective of the task sequence (A-B-A or C-B-A). Moreover, it follows that the performance should be similar to that for A-B-A sequences in the $n-2$ overlap condition, which was what we observed.

While our assumptions nicely account for the observed data, they do not explain why temporally overlapping cues and targets affected BI in this manner. Strictly speaking, our results are uninformative in this respect. However, we might offer some speculations. In several previous studies, BI was assumed to operate on task-shift trials in order to reduce interference from the previously relevant task set (Arbuthnott, 2005; Mayr \& Keele, 2000; Schuch \& Koch, 2003). According to these accounts, BI must be strong if the previous task set is still activated to a large extent (cf. Gade \& Koch, 2005), or if the activation differences between the relevant and irrelevant task sets are rather small (cf. Arbuthnott, 2005), or both. However, although it seems reasonable to assume that the strength of task-set inhibition depends on the (absolute and relative) strengths of task-set activation, the present results can not be explained along these lines for two reasons. First, as can be seen in Figure 3, the strength of inhibition in the A-B-A task sequences was the same irrespective of the temporal cue-target overlap on trial $n-2$. Second, if the different cuing conditions modulated the task-set activation levels differentially, then, in any case, this should have produced a main effect of $n-1$ temporal cue-target overlap on trial $n$. However, we did not observe such an effect.

Thus, the question is how else our effects can be explained. According to Arbuthnott (2005), symbol cues of the type applied here are relatively nonsalient, so that the tasks are difficult to discriminate. Consequently, in order to select the correct task set with reasonable accuracy, interference from both competing task sets must actually be reduced. Our results, though, indicate that with a temporal cue-target overlap on trial $n-2$, only the previously relevant task set is inhibited on trial $n-1$. Why should this be the case? First, one could assume that a strong association between the cue and the to-be-performed task is established on trial $n-2$ if the cues and targets overlap temporally. Such an association could then be used on trial $n-1$ to retrieve which task set was relevant on trial $n-2$, which allows selective inhibition. With respect to temporally nonoverlapping cues and targets on trial $n-2$, one could assume that they produce a weak cue-task association. It could therefore have been more difficult to remember on trial $n-1$ which task set was relevant on trial $n-2$. That is, since actually both irrelevant task sets produce interference on trial $n-1$ (cf. Arbuthnott, 2005), both are also subject to inhibition with nonoverlapping cues and targets on trial $n-2$.

A different account for our data would be to assume that there are two sources of interference in task switching, one cue-based and one task-set-based. ${ }^{2}$ With temporally overlapping cues and targets on trial $n-2$, the cue-based interference might primarily be relevant since the $n-2$ cue interferes with processing the $n-1$ cue (probably due to the only recent offset). Thus, inhibition is directed toward the $n-2$ cue, resulting in specific inhibition. The nonselective inhibition with temporally separated cues and targets on trial $n-2$ can then be explained as a consequence of task-set interference. That is, if interference occurs primarily at the task-set level (and not due to cue processing), then inhibition is no longer selective since both irrelevant task sets on trial $n-1$ interfere with performing the actually required task (due to their similar activation levels, cf. Arbuthnott, 2005).

Although these accounts explain the present data and are also in accord with previous results from other studies (Arbuthnott, 2005; Dreher \& Berman, 2002; Gade \& Koch, 2005), they nevertheless remain speculative. Further research, for instance by using a flanker paradigm similar to the one applied in Hübner et al. (2003), has to show whether it holds or whether alternatives are needed.

\section{AUTHOR NOTE}

This research was supported by a grant to the second author from the Deutsche Forschungsgemeinschaft (DFG: Hu 432/9). We thank Marco Steinhauser, W. Trammell Neill, Katherine Arbuthnott, and two anonymous reviewers for their helpful comments on the manuscript. Furthermore, we are grateful to Cindy Eckart and Johannes Schult for data acquisition. Correspondence relating to this article should be addressed to M. D. Druey, Universität Konstanz, Fachbereich Psychologie, Fach D29, D-78457 Konstanz, Germany (e-mail: michel.druey@uni-konstanz.de).

\section{REFERENCES}

Arbuthnott, K. D. (2005). The influence of cue type on backward inhibition. Journal of Experimental Psychology: Learning, Memory, \& Cognition, 31, 1030-1042.

ARBUTHNOTT, K. D., \& FrANK, J. (2000). Executive control in set switching: Residual switch cost and task-set inhibition. Canadian Journal of Experimental Psychology, 54, 33-41.

ARBUTHNOTT, K. D., \& WoODWARD, T. S. (2002). The influence of cuetask association and location on switch cost and alternating-switch cost. Canadian Journal of Experimental Psychology, 56, 18-29.

Dreher, J.-C., \& Berman, K. F. (2002). Fractionating the neural substrate of cognitive control processes. Proceedings of the National Academy of Sciences, 99, 14595-14600.

Dreher, J.-C., KohN, P. D., \& BERMAN, K. (2001). The neural basis of backward inhibition during task switching. NeuroImage, 13, 311.

GADE, M., \& КосH, I. (2005). Linking inhibition to activation in the control of task sequences. Psychonomic Bulletin \& Review, 12, 530-534.

Hübner, M., Dreisbach, G., Haider, H., \& Kluwe, R. H. (2003). Backward inhibition as a means of sequential task-set control: Evidence for reduction of task competition. Journal of Experimental Psychology: Learning, Memory, \& Cognition, 29, 289-297. 
Mayr, U. (2002). Inhibition of action rules. Psychonomic Bulletin \& Review, 9, 93-99.

MAYR, U., Diedrichsen, J., IvRY, R., \& KeEle, S. W. (2006). Dissociating task-set selection from task-set inhibition in the prefrontal cortex. Journal of Cognitive Neuroscience, 18, 14-21.

MAYR, U., \& KEELE, S. W. (2000). Changing internal constraints on action: The role of backward inhibition. Journal of Experimental Psychology: General, 129, 4-26.

MAYR, U., \& KLIEGL, R. (2003). Differential effects of cue changes and task changes on task-set selection costs. Journal of Experimental Psychology: Learning, Memory, \& Cognition, 29, 362-372.

MONSELl, S. (2003). Task switching. Trends in Cognitive Sciences, 7, 134-140.

RoGERS, R. D., \& Monsell, S. (1995). Costs of a predictable switch between simple cognitive tasks. Journal of Experimental Psychology: General, 124, 207-231.
ScHUCH, S., \& KocH, I. (2003). The role of response selection for inhibition of task sets in task shifting. Journal of Experimental Psychology: Human Perception \& Performance, 29, 92-105.

\section{NOTES}

1. We use the term temporal cue-target overlap in a descriptive way here. What we actually varied was the duration of cue presentation. Thus, this term does not imply that cues and targets are processed integratively, since temporal cue-target overlap and cue duration are confounded.

2. We are grateful to W. Trammell Neill for this suggestion.

(Manuscript received July 21, 2006; revision accepted for publication October 31,2006 .) 